\title{
Influence of Dietary Nitrate Supplementation on High-Intensity Intermittent Running Performance at Different Doses of Normobaric Hypoxia in Endurance-Trained Males
}

\author{
George P. Robinson, Sophie C. Killer, Zdravko Stoyanov, Harri Stephens, \\ Luke Read, Lewis J. James, and Stephen J. Bailey \\ Loughborough University
}

\begin{abstract}
This study investigated whether supplementation with nitrate-rich beetroot juice (BR) can improve high-intensity intermittent running performance in trained males in normoxia and different doses of normobaric hypoxia. Eight endurance-trained males $\left(\dot{\mathrm{V}} \mathrm{2}_{\text {2peak }}, 62 \pm 6 \mathrm{ml} \cdot \mathrm{kg}^{-1} \cdot \mathrm{min}^{-1}\right)$ completed repeated $90 \mathrm{~s}$ intervals at $110 \%$ of peak treadmill velocity, from an initial step incremental test, interspersed by $60 \mathrm{~s}$ of passive recovery until exhaustion $\left(T_{\mathrm{lim}}\right)$. Participants completed the first three experimental trials during days 3,5, and 7 of BR or nitrate-depleted beetroot juice (PLA) supplementation and completed the remaining experimental visits on the alternative supplement following at least 7 days of washout. The fraction of inspired oxygen during visits $1-3$ was either $0.209,0.182$, or 0.157 , equivalent to an altitude of $0,1,200$, and $2,400 \mathrm{~m}$, respectively, and this order was replicated on visits 4-6. Arterial oxygen saturation declined dose dependently as fraction of inspired oxygen was lowered $(p<.05)$. Plasma nitrite concentration was higher pre- and postexercise after BR compared with PLA supplementation $(p<.05)$. There was no difference in $\mathrm{T}_{\text {lim }}$ between PLA and BR at $0 \mathrm{~m}(445[324,508]$ and $410[368,548] \mathrm{s}) ; 1,200 \mathrm{~m}(341[270$, $390]$ and $332[314,356] \mathrm{s})$; or 2,400 $\mathrm{m}(233$ [177, 373] and 251 [221, 323] s) (median and [interquartile range]; $p>.05)$. The findings from this study suggest that short-term BR supplementation does not improve high-intensity intermittent running performance in endurance-trained males in normoxia or at doses of normobaric hypoxia that correspond to altitudes at which athletes typically train while on altitude training camps.
\end{abstract}

Keywords: beetroot juice, exercise performance, nitric oxide

Dietary supplementation with inorganic nitrate $\left(\mathrm{NO}_{3}^{-}\right)$, typically in the form of $\mathrm{NO}_{3}{ }^{-}$-rich beetroot juice (BR), has emerged as a nutritional intervention to enhance exercise performance (Jones et al., 2018). The ergogenic effects of dietary $\mathrm{NO}_{3}{ }^{-}$supplementation are attributed to the stepwise reduction of $\mathrm{NO}_{3}{ }^{-}$to nitrite $\left(\mathrm{NO}_{2}^{-}\right)$and then nitric oxide (NO), which can lead to improvements in skeletal muscle perfusion, metabolism, and contractile function (Jones et al., 2018). Initial studies indicated that dietary $\mathrm{NO}_{3}{ }^{-}$supplementation could improve continuous endurance performance in recreationally active or moderately trained individuals $\left(\dot{\mathrm{V} O}{ }_{2 \text { peak }}<60 \mathrm{ml} \cdot \mathrm{kg}^{-1} \cdot \mathrm{min}^{-1}\right.$; Bailey et al., 2009; Cermak et al., 2012; Porcelli et al., 2015; Wylie et al., 2013a), with subsequent studies revealing equivocal effects in well-trained endurance athletes $\left(\dot{\mathrm{V}}_{2 \text { peak }}>60 \mathrm{ml} \cdot \mathrm{kg}^{-1} \cdot \mathrm{min}^{-1}\right.$; Bescós et al., 2012; Boorsma et al., 2014; Bourdillon et al., 2015; Christensen et al., 2013; Porcelli et al., 2015; Rokkedal-Lausch et al., 2019; Shannon et al., 2017). More recently, $\mathrm{NO}_{3}{ }^{-}$supplementation has been reported to augment physiological processes, such as perfusion, calcium handling and contractility, to a greater extent in type II (fast-twitch), compared with type I (slow-twitch), skeletal muscle (Jones et al., 2016) and to improve high-intensity intermittent performance in moderately trained individuals (Aucouturier et al., 2015; Thompson et al., 2016; Wylie et al., 2013b, 2016) and trained

The authors are with the School of Sport, Exercise and Health Sciences, Loughborough University, Loughborough, Leicestershire, United Kingdom. Bailey (S.Bailey2@lboro.ac.uk) is corresponding author. team sport athletes (Nyakayiru et al., 2017). However, the effect of $\mathrm{NO}_{3}{ }^{-}$supplementation on high-intensity intermittent performance in endurance-trained athletes is equivocal (Bond et al., 2012; Christensen et al., 2013; Pawlak-Chaouch et al., 2019).

Since the reduction of $\mathrm{NO}_{2}^{-}$to $\mathrm{NO}$ is enhanced as $\mathrm{PO}_{2}$ declines (Jones et al., 2016), there has been great interest in the ergogenic potential of $\mathrm{NO}_{3}{ }^{-}$supplementation in hypoxia. In recreationally active or moderately trained individuals, $\mathrm{NO}_{3}{ }^{-}$supplementation has been reported to improve continuous endurance performance in normobaric hypoxia (Cocksedge et al., 2020; Kelly et al., 2014; Masschelein et al., 2012; Muggeridge et al., 2014), with greater improvements in hypoxia compared with normoxia (Cocksedge et al., 2020; Kelly et al., 2014). Although the effects of $\mathrm{NO}_{3}{ }^{-}$supplementation on continuous endurance performance in normobaric hypoxia in trained individuals are less clear (Arnold et al., 2015; Bourdillon et al., 2015; MacLeod et al., 2015; Nybäck et al., 2017; Rokkedal-Lausch et al., 2019), there is evidence to suggest that, in contrast to normoxia (Porcelli et al., 2015), improved performance with $\mathrm{NO}_{3}{ }^{-}$supplementation in hypoxia is not linked to aerobic fitness (Shannon et al., 2016). To date, we are only aware of one study to have assessed the effect of $\mathrm{NO}_{3}{ }^{-}$ supplementation on repeated sprint cycling performance (four sets of $9 \times 4 \mathrm{~s}$ ) in normobaric hypoxia, with this study reporting no effect on peak or mean power output throughout the test, but a reduction in work decrement with $\mathrm{BR}$ supplementation in the first set (Kent et al., 2019). While this suggests that $\mathrm{NO}_{3}{ }^{-}$supplementation could confer some ergogenic effect for team sports athletes in hypoxia, the effect of $\mathrm{NO}_{3}{ }^{-}$supplementation on high-intensity 
intermittent performance in endurance-trained athletes in hypoxia has yet to be determined. This is important to resolve as highintensity interval training is a common training method employed by endurance athletes at altitude, and if $\mathrm{NO}_{3}{ }^{-}$supplementation was able to improve high-intensity exercise performance in hypoxia, this could translate into improved training quality while at terrestrial altitude training camps.

Heretofore, laboratory studies reporting improved performance following $\mathrm{NO}_{3}{ }^{-}$supplementation in normobaric hypoxia have administered gas mixtures with a fraction of inspired oxygen $\left(\mathrm{FIO}_{2}\right)$ equivalent to moderate-to-high altitude $(\geq 2,500 \mathrm{~m})$. However, it is well known that most athletes complete training sessions while at terrestrial altitude training camps at a low-to-moderate altitude $(<2,500 \mathrm{~m})$ and that further research is required to assess the ergogenic potential of different dietary interventions at such altitudes (Stellingwerff et al., 2019). Therefore, the potential translation of findings from laboratory studies assessing the effect of $\mathrm{NO}_{3}{ }^{-}$supplementation on performance in hypoxia to performance at altitude training camps is unclear, particularly for highintensity intermittent exercise performance which has received comparatively little attention. The purpose of the current study was to assess the effect of short-term BR supplementation on highintensity intermittent running performance in normoxia and at doses of normobaric hypoxia equivalent to terrestrial altitudes most frequently used during training by British Olympic middle distance and endurance athletes (1,200 and 2,400 $\mathrm{m}$ ) and as studied previously (Chapman et al., 2014). It is well documented that arterial and muscle oxygen saturation decline dose dependently as the hypoxic dose is increased (Goodall et al., 2010). Since the improvements in performance following BR supplementation in hypoxia are greatest in those presenting with lower muscle oxygenation (Cocksedge et al., 2020), it was hypothesized that BR supplementation would progressively enhance high-intensity intermittent running performance as the hypoxic dose was increased.

\section{Methods}

\section{Participants}

Eight endurance-trained males (mean $\pm S D$ : age $23 \pm 4$ years, stature $1.77 \pm 0.04 \mathrm{~m}$, body mass $\left.69 \pm 7 \mathrm{~kg}, \dot{\mathrm{VO}}{ }_{2 \text { peak }} 62 \pm 6 \mathrm{ml} \cdot \mathrm{kg}^{-1} \cdot \mathrm{min}^{-1}\right)$, familiar with intense intermittent running, volunteered to participate in this study. The participants were middle- or long-distance runners or triathletes who were completing at least four weekly endurance sessions (high-intensity interval training or submaximal continuous training). The study procedures were approved by the Loughborough University Research Ethics Approvals Human Participants Sub-Committee, and all participants were required to give their written informed consent prior to commencement of the study after the experimental procedures, associated risks, and benefits of participation had been explained in detail. Participants were instructed to avoid alcohol and strenuous exercise in the $24 \mathrm{hr}$ preceding each test and caffeine intake $6 \mathrm{hr}$ prior to each test. Participants were also instructed to abstain from using antibacterial mouthwash throughout the study as this interferes with oral $\mathrm{NO}_{3}{ }^{-}$-reducing bacteria.

\section{Experimental Overview}

Participants were required to report to the laboratory on eight occasions over a 4 week period with the eight visits separated by at least $48 \mathrm{hr}$. Following the completion of preliminary exercise tests (Visits 1-2), arterial $\mathrm{O}_{2}$ saturation $\left(\mathrm{SpO}_{2}\right)$, plasma $\left[\mathrm{NO}_{2}{ }^{-}\right]$, and time to exhaustion $\left(T_{\text {lim }}\right)$ were assessed during a high-intensity intermittent running protocol completed in six experimental conditions (Visits 3-8). These conditions consisted of three different $\mathrm{FIO}_{2}$ values $(0.209,0.182$, and 0.157 , equivalent to terrestrial altitudes of sea level [0 m], 1,200 m, and 2,400 m, respectively) completed following two different dietary supplementation strategies (BR and $\mathrm{NO}_{3}{ }^{-}$-depleted beetroot juice [PLA]). The study employed a repeated-measures, crossover design. The PLA and BR supplements were administered double blind in a randomized balanced order (four participants received BR first). The order of $\mathrm{FIO}_{2}$ conditions was administered single blind and randomized for the first supplementation condition, with this order reproduced for the second supplementation condition. A schematic of the experimental design is illustrated in Figure 1.

\section{Preliminary Tests}

On the first laboratory visit, participants completed a step incremental running test on a motorized treadmill (Excite MED; Technogym UK Ltd., Bracknell, United Kingdom) for determination of $\dot{\mathrm{V}} \mathrm{O}_{2 \text { peak }}$ and peak treadmill velocity (PTV). The test commenced at $12-14 \mathrm{~km} / \mathrm{hr}$ and comprised a series of $3 \mathrm{~min}$ stages with the treadmill velocity increased by $1 \mathrm{~km} / \mathrm{hr}$ at the end of each stage and the treadmill gradient was maintained at $1 \%$ throughout the test. The test was terminated when the participants dismounted the treadmill and $T_{\lim }$ was recorded. The PTV was calculated as follows and as described by Bosquet et al. (2007):

$$
\mathrm{PTV}=V+[I \times(T / S)],
$$

where $V$ is the velocity (in $\mathrm{km} / \mathrm{hr}$ ) of the last completed stage, $I$ is the increment of the test (in $\mathrm{km} / \mathrm{hr}$ ), $T$ is the duration (in seconds) maintained after the first $60 \mathrm{~s}$ of the final stage, and $S$ is the duration (in seconds) of the stage. Throughout the test, participants breathed through a low dead-space $(89 \mathrm{ml}$ ) facemask (Hans Rudolph; Cranlea, Birmingham, United Kingdom) with a two-way nonrebreathing valve system (2700 Series; Hans Rudolph) which was connected, via an outlet tube, to a series of Douglas bags (Harvard Biosciences Inc., Cambridge, United Kingdom). Expired air was collected into Douglas bags during the last minute of each stage and during the minute prior to exhaustion, as indicated by the participant, and analyzed for oxygen and carbon dioxide concentrations (Servomex 1400 Oxygen and Carbon Dioxide Gas Analyser; Servomex, Crowborough, United Kingdom). Gas volume (Harvard dry gas meter, Harvard Apparatus Ltd., Edenbridge, Cambridge, United Kingdom) and temperature (RS Pro digital thermometer; RS Components, Corby, United Kingdom) were measured and corrected to standard temperature and pressure, dry.

On the second visit to the laboratory, participants completed a high-intensity intermittent running protocol continued until $T_{\text {lim }}$ in normobaric hypoxia $(1,200 \mathrm{~m})$ for familiarization with the exercise protocol and all experimental procedures described below.

\section{Supplementation Procedures}

Following completion of the preliminary tests, participants were assigned to receive $140 \mathrm{ml}$ of $\mathrm{BR}$ (providing $\sim 12.4 \mathrm{mmol} \mathrm{NO}_{3}{ }^{-}$) or PLA (providing $\sim 0.08 \mathrm{mmol} \mathrm{NO}_{3}{ }^{-}$) (Beet it; James White Drinks Ltd., Ipswich, United Kingdom) daily for 7 days. On nonexperimental days (days 1-2, 4, and 6) during each supplementation period, participants consumed $1 \times 70 \mathrm{ml}$ in the morning $(\sim 09: 00)$ and $1 \times 70 \mathrm{ml}$ in the evening ( 19:00). On experimental days (days 3,5 , and 7 of supplementation), participants consumed $2 \times 70 \mathrm{ml} 2.5 \mathrm{hr}$ before arriving at the laboratory ( $3 \mathrm{hr}$ prior to 
Visit 1: Step incremental test

Visit 2: High-intensity intermittent

familiarization test
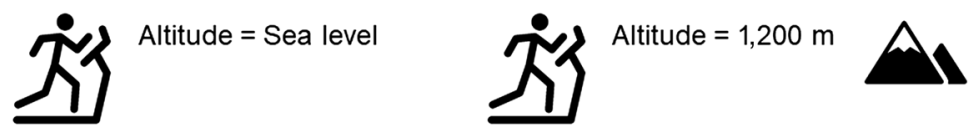

Visits 3-5: High-intensity intermittent test

Visits 6-8: High-intensity intermittent test
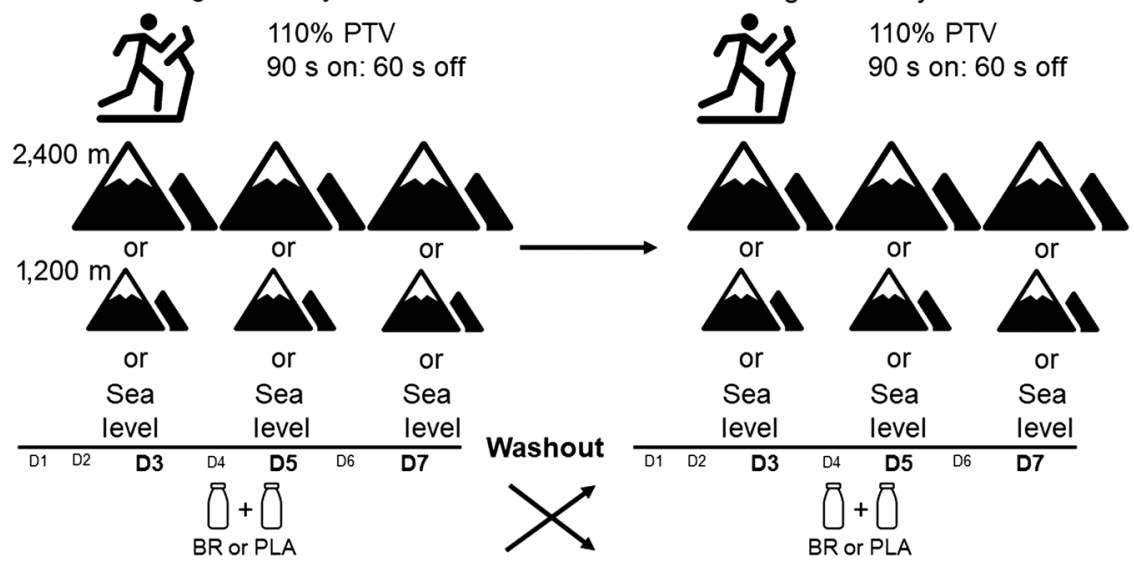

Figure 1 - A schematic of the experimental protocol. The sea level (0), 1,200, and 2,400 $\mathrm{m}$ values are the terrestrial altitudes equivalent to the FIO conditions $\left(0.209,0.182\right.$, and 0.157 , respectively) administered in the current study. $\mathrm{FIO}_{2}=$ fraction of inspired oxygen; BR = nitrate-rich beetroot juice; $\mathrm{D}=$ day; PLA = nitrate-depleted beetroot juice; PTV = peak treadmill velocity.

commencing the high-intensity intermittent running test) to coincide with peak plasma $\left[\mathrm{NO}_{2}^{-}\right]$(Wylie et al., 2013a). A washout period of at least 7 days separated the first and second supplementation periods, after which participants began supplementing with the alternative supplement.

\section{Experimental Trials}

Participants were instructed to record their diet in the $48 \mathrm{hr}$ preceding their first experimental trial and to replicate this diet $48 \mathrm{hr}$ prior to all subsequent trials. After arriving at the laboratory, participants rested in a supine position for $10 \mathrm{~min}$ while breathing normoxic room air. During this period, $\mathrm{SpO}_{2}$ was continually monitored (Anapulse 100; ANA WIZ Ltd., Surrey, United Kingdom) and reported as the mean value over the final $3 \mathrm{~min}$. A $6 \mathrm{ml}$ venous blood sample from a forearm vein was then collected into a lithium heparin tube and centrifuged at $3,500 \times g$ for $10 \mathrm{~min}$ at $4{ }^{\circ} \mathrm{C}$. The plasma was aliquoted into $1.5 \mathrm{ml}$ microcentrifuge tubes and stored at $-80^{\circ} \mathrm{C}$ until later analysis of plasma $\left[\mathrm{NO}_{2}{ }^{-}\right]$using ozonebased chemiluminescence as described previously (Wylie et al., 2013a). Participants were then fitted with a facemask with the inlet of the two-way nonrebreathing valve system connected to a series of Douglas bags which contained gas mixtures with an $\mathrm{FIO}_{2}$ of $0.209,0.182$, or 0.157 . The inspirates were generated using an altitude simulation generator (Everest Summit II Altitude Generator; Hypoxico Inc., New York, NY). The $\mathrm{FIO}_{2}$ of the gas mixtures was verified prior to each test using a calibrated Servomex 1400 Oxygen and Carbon Dioxide Gas Analyser. Participants inhaled the allocated gas mixture for the remainder of the trial until the exhaustive high-intensity intermittent running protocol had been completed. Initially, participants remained supine during a 10-min wash-in period with $\mathrm{SpO}_{2}$ recorded every minute and reported as the mean value over the final $3 \mathrm{~min}$ of wash-in.

After completing the gas wash-in period, participants transferred to the treadmill where they straddled the treadmill belt before completing a 5-min warm-up at a velocity corresponding to $60 \%$ PTV. Participants then straddled the treadmill belt for a $2 \mathrm{~min}$ recovery before commencing the exhaustive high-intensity intermittent running protocol. The protocol comprised repeated $90 \mathrm{~s}$ intervals at a velocity corresponding to $110 \%$ PTV, interspersed with $60 \mathrm{~s}$ passive recovery straddling the treadmill belt. This velocity was selected as it is $\sim 5 \%$ lower than $800-\mathrm{m}$ race pace (Bosquet et al., 2007) and would broadly reflect the velocity at which middle distance runners would complete a high-quality high-intensity intermittent training session. Using the $T_{\text {lim }}$ data from the 1,200-m familiarization trial and the 1,200-m PLA trial, the coefficient of variation for the high-intensity intermittent running test administered in the current study was $7 \%$. Prior to the start of each interval, participants were provided with a $3 \mathrm{~s}$ countdown and began lowering themselves onto the treadmill belt on the count of three to ensure they were fully running after $60 \mathrm{~s}$ of recovery. The test was terminated when participants dismounted the treadmill and $T_{\text {lim }}$ was recorded. After completing the test, the facemask was removed, and participants breathed normoxia room air while lying supine. Following a 5-min recovery, a venous blood sample was obtained and centrifuged for later determination of plasma $\left[\mathrm{NO}_{2}{ }^{-}\right]$(as described previously). The rate of change in plasma $\left[\mathrm{NO}_{2}^{-}\right]$was calculated as the change in plasma $\left[\mathrm{NO}_{2}{ }^{-}\right]$ $(\mathrm{nM}) / T_{\lim }(\min )$.

\section{Statistical Analysis}

Statistical analysis was performed using IBM SPSS Statistics (version 25, Armonk, NY). Initially, Shapiro-Wilk tests were used to check data normality. Plasma $\left[\mathrm{NO}_{2}{ }^{-}\right]$was analyzed using a 6 (condition) $\times 2$ (time) repeated-measures ANOVA with effects size (ES) calculated using partial eta squared $\left(\eta_{\mathrm{p}}^{2}\right)$. Where the ANOVA revealed a significant effect, paired $t$ tests with Fisher's LSD correction were utilized to determine the origin of any effects with ES calculated as Cohen's $d_{\mathrm{z}}(t / \sqrt{ } n)$. The rate of change 
in plasma $\left[\mathrm{NO}_{2}^{-}\right], T_{\text {lim }}$, and $\mathrm{SpO}_{2}$ data were analyzed using Friedman's ANOVA. Where Friedman's ANOVA revealed a significant effect, Wilcoxon signed-rank tests were used to assess the origin of any effects with ES calculated as $Z / \sqrt{ } n$. Spearman's rank $\left(r_{\mathrm{s}}\right)$ was used to test for correlations between intercondition differences in plasma $\left[\mathrm{NO}_{2}{ }^{-}\right]$and $T_{\text {lim }}$, and intercondition differ-

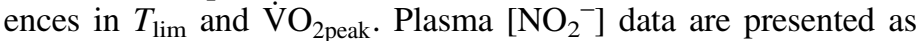
mean $\pm S D$ and the rate of change in plasma $\left[\mathrm{NO}_{2}^{-}\right], T_{\text {lim }}$, and $\mathrm{SpO}_{2}$ data are presented as median and interquartile range (25th and 75 th percentiles) unless otherwise stated. Statistical significance was accepted at $p<.05$.

\section{Results}

\section{$\mathrm{SpO}_{2}$}

Friedman's ANOVA indicated significant intercondition differences in $\mathrm{SpO}_{2}(p<.05$; Table 1$)$. There was no pre-post wash-in difference in $\mathrm{SpO}_{2}$ with $\mathrm{BR}$ or PLA supplementation at $0 \mathrm{~m}$ $(p>.05)$. Post wash-in $\mathrm{SpO}_{2}$ was lower at 1,200 m compared with $0 \mathrm{~m}(\mathrm{ES}=0.72$ and 0.67$)$, and $2,400 \mathrm{~m}$ compared with $1,200 \mathrm{~m}(\mathrm{ES}=0.70$ and 0.78$)$ and $0 \mathrm{~m}(\mathrm{ES}=0.89$ and 0.84$)$ following both PLA and BR supplementation $(p<.05)$. There were no differences between PLA and BR for $\mathrm{SpO}_{2}$ at $0,1,200$ or $2,400 \mathrm{~m}(p>.05)$.

\section{Plasma $\left[\mathrm{NO}_{2}^{-}\right]$}

There were significant main effects for condition $(E S=0.86)$ and time $(\mathrm{ES}=0.76)$ and a condition $\times$ time interaction effect $(\mathrm{ES}=0.68)$ for plasma $\left[\mathrm{NO}_{2}{ }^{-}\right](p<.05)$. Preexercise $(\mathrm{ES}=2.22-2.65)$ and postexercise $(\mathrm{ES}=0.80-0.89)$ plasma $\left[\mathrm{NO}_{2}^{-}\right]$were higher following $\mathrm{BR}$ compared with PLA supplementation $(p<.05$; Table 2; Figure 2$)$.
Postexercise plasma $\left[\mathrm{NO}_{2}^{-}\right]$was not different from pre-exercise plasma $\left[\mathrm{NO}_{2}^{-}\right]$following PLA supplementation $(\mathrm{ES}=0.19-0.45$; $p>.05)$ but was lower than preexercise plasma $\left[\mathrm{NO}_{2}^{-}\right]$following BR supplementation ( $\mathrm{ES}=1.32-1.69 ; p>.05)$. Friedman's ANOVA indicated significant intercondition differences in the rate of change in plasma $\left[\mathrm{NO}_{2}^{-}\right](p<.05)$. Compared with PLA, the rate of change in plasma $\left[\mathrm{NO}_{2}^{-}\right]$was greater following $\mathrm{BR}$ supplementation $(\mathrm{ES}=$ $0.84-0.89 ; p<.05)$. There was no difference in the rate of change in plasma $\left[\mathrm{NO}_{2}^{-}\right]$between the PLA trials $(\mathrm{ES}=0.09-0.17)$ or between the BR trials at 0 and $1,200 \mathrm{~m}(\mathrm{ES}=0.22)(p>.05)$, but plasma $\left[\mathrm{NO}_{2}{ }^{-}\right]$declined at a greater rate in the $\mathrm{BR}$ trial at $2,400 \mathrm{~m}$ compared with $0 \mathrm{~m}(\mathrm{ES}=0.79)$ and $1,200 \mathrm{~m}(\mathrm{ES}=0.74)(p<.05$; Table 2$)$.

\section{$T_{\lim }$}

The $110 \%$ PTV that was administered in the high-intensity intermittent running test was $19 \pm 1 \mathrm{~km} / \mathrm{hr}$. Friedman's ANOVA indicated significant intercondition differences in $T_{\lim }(p<.05$; Figure 3 ). Compared with $0 \mathrm{~m}, T_{\text {lim }}$ was lowered dose dependently in the 1,200 and $2,400 \mathrm{~m}$ conditions $(p<.05)$. There were no differences in $T_{\text {lim }}$ between PLA and BR supplementation at $0 \mathrm{~m}$ $(445$ [324, 508] and 410 [368, 548] s; ES = 0.26), 1,200 m (341 $[270,390]$ and $332[314,356] \mathrm{s}$; ES $=0.06)$ or $2,400 \mathrm{~m}(233$ [177, $373]$ and $251[221,323] \mathrm{s} ; \mathrm{ES}=0.12)(p>.05)$. The change in $T_{\text {lim }}$ between the PLA and BR supplementation conditions at $0 \mathrm{~m}$ $\left(r_{\mathrm{s}}=-.62\right), 1,200 \mathrm{~m}\left(r_{\mathrm{s}}=.25\right)$ and $2,400 \mathrm{~m}\left(r_{\mathrm{s}}=.17\right)$ was not correlated with $\mathrm{VO}_{2 \text { peak }}(p>.05)$. Moreover, the change in $T_{\text {lim }}$ between the PLA and BR conditions was not correlated with the difference in preexercise plasma $\left[\mathrm{NO}_{2}{ }^{-}\right]$between PLA and $\mathrm{BR}$, or the change in plasma $\left[\mathrm{NO}_{2}{ }^{-}\right]$pre- to postexercise in the BR conditions, at $0 \mathrm{~m}\left(r_{\mathrm{s}}=.41\right.$ and -.59$), 1,200 \mathrm{~m}\left(r_{\mathrm{s}}=.41\right.$ and -.71$)$, or $2,400 \mathrm{~m}\left(r_{\mathrm{s}}=.14\right.$ and -.07$)(p \geq .05)$. However, the change in $T_{\text {lim }}$ between the PLA and BR and the change in plasma $\left[\mathrm{NO}_{2}^{-}\right]$pre- to

Table 1 Arterial Oxygen Saturation Responses Prior to and at the End of a Resting Wash-in Inhalation Period With Different Normobaric Gas Mixtures After Supplementation With BR and PLA

\begin{tabular}{|c|c|c|c|c|c|c|}
\hline & \multicolumn{2}{|c|}{$0 \mathrm{~m}$} & \multicolumn{2}{|c|}{$1,200 \mathrm{~m}$} & \multicolumn{2}{|c|}{$2,400 \mathrm{~m}$} \\
\hline & PLA & BR & PLA & BR & PLA & BR \\
\hline Pre $(\%)$ & $99(98,99)$ & $99(98,99)$ & $99(98,99)$ & $99(98,99)$ & $99(98,99)$ & $99(98,99)$ \\
\hline End $(\%)$ & $99(98,99)$ & $99(98,99)$ & $97(95,99)^{*}$ & $97(95,99)^{*}$ & $96(94,96)^{* *}$ & $96(93,97)^{* * *}$ \\
\hline
\end{tabular}

Note. Data are presented as median and interquartile range (25th and 75 th percentile). The $0,1,200$, and 2,400 $\mathrm{m}$ values are the terrestrial altitudes equivalent to the FIO ${ }_{2}$ conditions $(0.209,0.182$, and 0.157 , respectively $)$ administered in the current study. $\mathrm{BR}=$ nitrate-rich beetroot juice; $\mathrm{PLA}=$ nitrate-depleted beetroot juice; FIO ${ }_{2}=$ fraction of inspired oxygen.

*Significantly different from pre and the corresponding point in the $0 \mathrm{~m}$ condition $(p<.05){ }^{* * *}$ Significantly different from pre and the corresponding points in the 0 and 1,200 m conditions $(p<.05)$.

Table 2 Plasma Nitrite Concentration Responses During a High-Intensity Intermittent Running Protocol Completed While Inhaling Different Normobaric Gas Mixtures After Supplementation With BR and PLA

\begin{tabular}{|c|c|c|c|c|c|c|}
\hline & \multicolumn{2}{|c|}{$0 \mathrm{~m}$} & \multicolumn{2}{|c|}{$1,200 \mathrm{~m}$} & \multicolumn{2}{|c|}{$2,400 \mathrm{~m}$} \\
\hline & PLA & BR & PLA & BR & PLA & BR \\
\hline Pre $(\mathrm{nM})$ & $96 \pm 39$ & $384 \pm 136^{*}$ & $84 \pm 23$ & $342 \pm 105^{*}$ & $82 \pm 22$ & $386 \pm 138^{*}$ \\
\hline Post (nM) & $89 \pm 27$ & $207 \pm 47^{* *}$ & $75 \pm 18$ & $185 \pm 52^{* *}$ & $76 \pm 18$ & $178 \pm 55^{* *}$ \\
\hline Rate of change (nM/min) & $1(-6,0)$ & $-24(-39,-9)^{*}$ & $-3(-5,1)$ & $-25(-40,-10)^{*}$ & $-0.5(-9,2)$ & $-41(-55,-27)^{* * *}$ \\
\hline
\end{tabular}

Note. Pre and post data are presented as mean $\pm S D$ with rate of change data presented as median and interquartile range (25th and 75 th percentile). The $0,1,200$, and $2,400 \mathrm{~m}$ values are the terrestrial altitudes equivalent to the $\mathrm{FIO}_{2}$ conditions $(0.209,0.182$, and 0.157 , respectively) administered in the current study. $\mathrm{BR}=$ nitrate-rich beetroot juice; PLA = nitrate-depleted beetroot juice; $\mathrm{FIO}_{2}=$ fraction of inspired oxygen .

*Significantly different from PLA $(p<.05)$. ${ }^{* *}$ Significantly different from PLA and BR pre $(p<.05)$. ${ }^{* * *}$ Significantly different from PLA, BR $0 \mathrm{~m}$, and BR $1,200 \mathrm{~m}$ $(p<.05)$. 


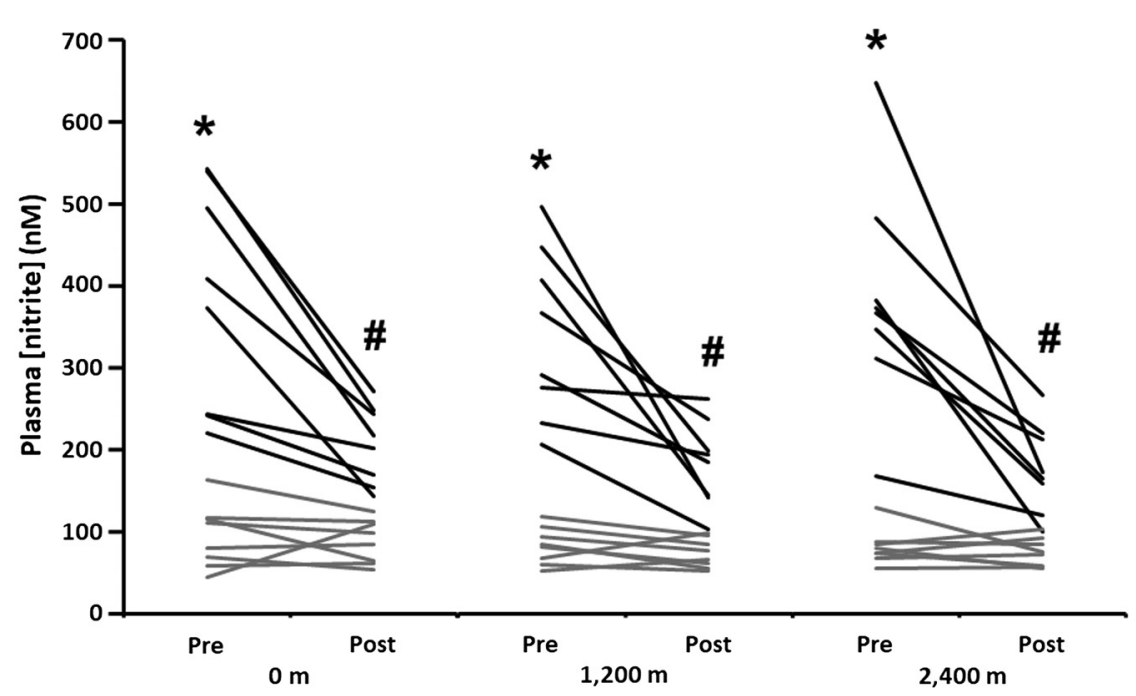

Figure 2 - Plasma nitrite concentration ([nitrite]) at rest (pre) and after (post) a high-intensity intermittent running protocol continued until exhaustion while inhaling different normobaric gas mixtures. Data are individual responses with black lines representing responses following BR supplementation and gray lines representing responses following PLA supplementation. The $0,1,200$, and $2,400 \mathrm{~m}$ values are the terrestrial altitudes equivalent to the FIO ${ }_{2}$ conditions $(0.209,0.182$, and 0.157 , respectively) administered in the current study. *Significantly different from pre in the PLA condition $(p<.05)$. ${ }^{\#}$ Significantly different from pre in the BR condition, and pre and post in the PLA conditions $(p<.05)$.

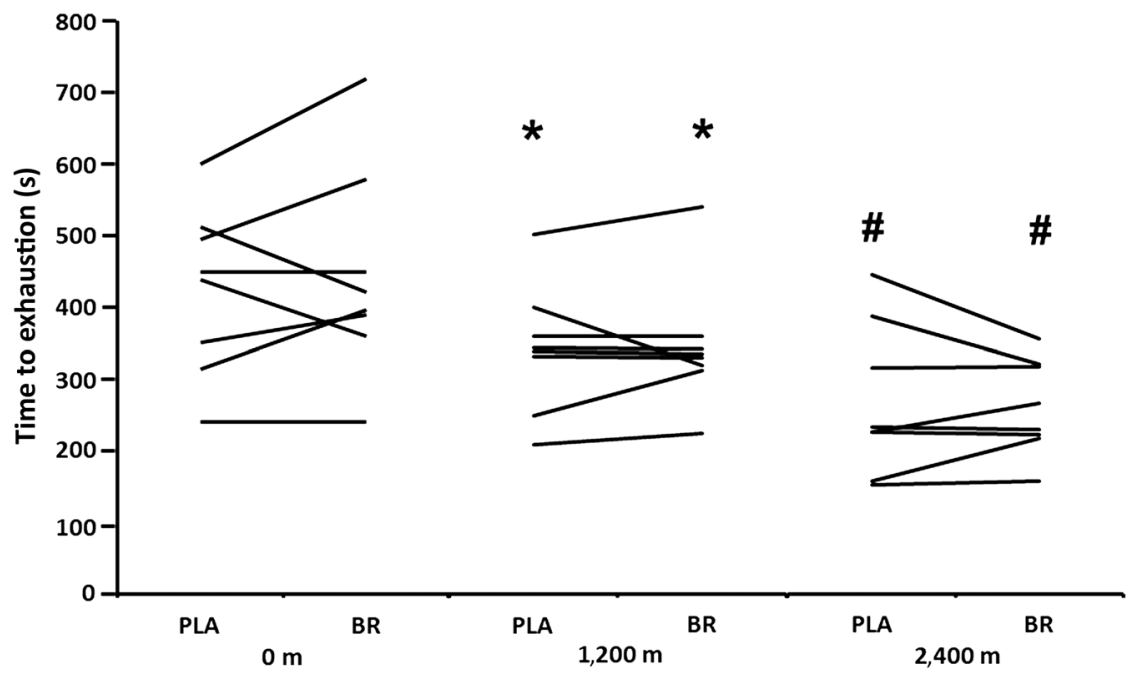

Figure 3 - Time to exhaustion during a high-intensity intermittent running protocol continued until exhaustion while inhaling different normobaric gas mixtures. Data are individual responses following supplementation with BR and PLA. The 0,1,200, and 2,400 m values are the terrestrial altitudes equivalent to the $\mathrm{FIO}_{2}$ conditions $(0.209,0.182$, and 0.157 , respectively) administered in the current study. $\mathrm{BR}=$ nitrate-rich beetroot juice; $\mathrm{PLA}=$ nitratedepleted beetroot juice; $\mathrm{FIO}_{2}=$ fraction of inspired oxygen. ${ }^{*}$ Significantly different from BR and PLA in the $0 \mathrm{~m}$ condition $(p<.05)$. ${ }^{\#}$ Significantly different from BR and PLA in the 0 and $1,200 \mathrm{~m}$ conditions $(p<.05)$.

postexercise following BR supplementation at $1,200 \mathrm{~m}\left(r_{\mathrm{s}}=-.71\right.$, $p=.05)$, and when data from all $\mathrm{FIO}_{2}$ conditions were combined $\left(r_{\mathrm{s}}=-.38, p=.066\right)$, approached statistical significance.

\section{Discussion}

The principal original findings of this study were that short-term BR supplementation, which increased plasma $\left[\mathrm{NO}_{2}{ }^{-}\right]$, did not improve high-intensity intermittent running performance in normoxia or low-to-moderate doses of normobaric hypoxia in trained males. These findings conflict with our experimental hypothesis and do not support an ergogenic effect of short-term BR supplementation in normoxia or doses of normobaric hypoxia equivalent to the altitudes that endurance athletes train at during altitude training camps. Therefore, it appears that BR supplementation is unlikely to improve high-intensity interval training quality at altitude training camps in endurance-trained males.

There was a dose-dependent lowering in $\mathrm{SpO}_{2}$ in the present study as the $\mathrm{FIO}_{2}$ of the administered gas mixture was reduced. This observation is consistent with previous work, which has also reported a dose-dependent decrease in muscle oxygenation (Goodall et al., 2010). In addition, resting plasma $\left[\mathrm{NO}_{2}{ }^{-}\right]$was 
increased by a comparable magnitude across the BR trials and with previous studies administering a similar dose of BR (Kelly et al., 2014; Nyakayiru et al., 2017; Shannon et al., 2016; Thompson et al., 2016; Wylie et al., 2016). Therefore, the manipulation of $\mathrm{FIO}_{2}$ in the present study was successful at lowering systemic oxygenation, and supplementation with $\mathrm{BR}$ was effective at increasing plasma $\left[\mathrm{NO}_{2}^{-}\right]$as a source of $\mathrm{O}_{2}$-independent $\mathrm{NO}$ generation.

Plasma $\left[\mathrm{NO}_{2}^{-}\right]$was lowered by a similar magnitude postexercise compared with preexercise across the BR trials in normoxia and both hypoxic doses, with postexercise plasma $\left[\mathrm{NO}_{2}{ }^{-}\right]$ higher in the BR compared with the PLA trials. These observations are consistent with previous reports of a similar decline in plasma $\left[\mathrm{NO}_{2}{ }^{-}\right]$during exhaustive exercise in normoxia and hypoxia (Cocksedge et al., 2020; Kelly et al., 2014) and suggest that circulating $\mathrm{NO}_{2}{ }^{-}$is used as a substrate and reduced to bioactive NO. Interestingly, the rate of decline in plasma $\left[\mathrm{NO}_{2}{ }^{-}\right]$during the exhaustive high-intensity intermittent running protocol was similar after BR supplementation in the 0 and 1,200-m conditions but was greater than both these conditions in 2,400 $\mathrm{m}$. This novel observation suggests that the greater plasma $\mathrm{NO}_{2}{ }^{-}$pool after BR supplementation is only used more rapidly during exhaustive intermittent exercise after moderate, but not low, hypoxic conditions compared with normoxia. However, it should be acknowledged that since the hypoxic gas administration was ceased as soon as participants attained exhaustion and before the postexercise blood sample was collected, this could have impacted the postexercise plasma $\left[\mathrm{NO}_{2}^{-}\right]$data.

Despite increasing plasma $\left[\mathrm{NO}_{2}{ }^{-}\right], \mathrm{BR}$ supplementation did not improve $T_{\text {lim }}$ during the high-intensity intermittent running protocol in normoxia in trained endurance athletes. These observations are consistent with most (Christensen et al., 2013; Muggeridge et al., 2013; Pawlak-Chaouch et al., 2019), but not all (Bond et al., 2012), studies in well-trained endurance athletes, and with findings that the ergogenic potential of BR supplementation declines as aerobic fitness $\left(\dot{\mathrm{V}} \mathrm{O}_{2 \text { peak }}\right)$ increases (Porcelli et al., 2015). Since type I muscle fibers appear less responsive to $\mathrm{NO}_{3}{ }^{-}$supplementation than type II muscle fibers (Jones et al., 2016), the blunted ergogenic effect of BR supplementation in well-trained endurance athletes might be linked to the large proportion of type I muscle fibers manifest in such individuals. Similarly, BR supplementation did not improve high-intensity intermittent running performance at low $(2,400 \mathrm{~m})$ and moderate $(1,200 \mathrm{~m})$ hypoxic doses in the current study. Although one previous study has reported BR supplementation to be largely ineffective at improving repeated sprint cycling performance (four sets of $9 \times 4 \mathrm{~s}$ ) in normobaric hypoxia (equivalent to $3,200 \mathrm{~m}$ altitude) in moderately trained individuals (Kent et al., 2019), which might have implications for team sports athletes competing in hypoxia, the relevance of this finding for endurance athletes completing high-intensity interval training sessions at altitude is likely to be limited. The original findings from the present study extend the previous observations by Kent et al. (2019) to indicate that BR supplementation does not improve high-intensity intermittent running performance during low-to-moderate hypoxia in well-trained athletes. It is possible that the improvements in muscle blood flow and capillarization with chronic endurance training permitted better maintenance of muscle oxygenation and lowered the reduction of circulating $\mathrm{NO}_{2}{ }^{-}$to $\mathrm{NO}$ in the endurancetrained males who participated in the current study (Jones et al., 2016, 2018). In turn, this may have limited the scope for an ergogenic effect of BR supplementation during high-intensity intermittent running.
It is acknowledged that a limitation of the present study was the small sample size. However, given the small effect size of BR on high-intensity intermittent running performance in this study, it seems unlikely that our conclusions would be altered by a larger sample size. It is also acknowledged that, since we used a group of endurance-trained males, our findings might not necessarily translate to endurance-trained females (Wickham \& Spriet, 2019).

In conclusion, while BR supplementation increased plasma $\left[\mathrm{NO}_{2}{ }^{-}\right]$and thus provided greater substrate for $\mathrm{O}_{2}$-independent $\mathrm{NO}$ synthesis during exercise, this did not translate into improved highintensity intermittent running performance in normoxia or low-tomoderate doses of hypoxia in endurance-trained males. These original findings further understanding of the ergogenic potential of BR supplementation at hypoxic doses comparable with those that would be encountered during training sessions at altitude training camps $(1,200-2,400 \mathrm{~m})$. The observations from the current study do not support BR supplementation as a dietary intervention to enhance high-intensity intermittent running performance in normoxia or low-to-moderate doses of hypoxia in endurancetrained males.

\section{Acknowledgments}

The study was designed by G.P. Robinson, S.C. Killer, L.J. James, and S.J. Bailey; data were collected and analyzed by G.P. Robinson, Z. Stoyanov, H. Stephens, L. Read, and S.J. Bailey; data interpretation and manuscript preparation were undertaken by G.P. Robinson, S.C. Killer, L.J. James, and S.J. Bailey. All authors approved the final version of the paper. The authors are grateful to Lewis Taylor, Sam Brazier, Alice Ashe, and Rory Mallace for assistance with data collection. This research was supported by a research grant from U.K. Athletics and English Institute of Sport to S.J. Bailey. In addition, G.P. Robinson was supported by a Rank Prize Funds COVID-19 Disruption Response Award.

\section{References}

Arnold, J.T., Oliver, S.J., Lewis-Jones, T.M., Wylie, L.J., \& Macdonald, J.H. (2015). Beetroot juice does not enhance altitude running performance in well-trained athletes. Applied Physiology Nutrition and Metabolism, 40(6), 590-595. doi:10.1139/apnm-2014-0470

Aucouturier, J., Boissière, J., Pawlak-Chaouch, M., Cuvelier, G., \& Gamelin, F.X. (2015). Effect of dietary nitrate supplementation on tolerance to supramaximal intensity intermittent exercise. Nitric Oxide, 49, 16-25. PubMed ID: 26028570 doi:10.1016/j.niox.2015.05.004

Bailey, S.J., Winyard, P., Vanhatalo, A., Blackwell, J.R., Dimenna, F.J., Wilkerson, D.P., . . J Jones, A.M. (2009). Dietary nitrate supplementation reduces the $\mathrm{O}_{2}$ cost of low-intensity exercise and enhances tolerance to high-intensity exercise in humans. Journal of Applied Physiology, 107(4), 1144-1155. PubMed ID: 19661447 doi:10.1152/ japplphysiol.00722.2009

Bescós, R., Ferrer-Roca, V., Galilea, P.A., Roig, A., Drobnic, F., Sureda, A., ... Pons, A. (2012). Sodium nitrate supplementation does not enhance performance of endurance athletes. Medicine \& Science in Sports \& Exercise, 44(12), 2400-2409. PubMed ID: 22811030 doi:10.1249/MSS.0b013e3182687e5c

Bond, H., Morton, L., \& Braakhuis, A.J. (2012). Dietary nitrate supplementation improves rowing performance in well-trained rowers. International Journal Sports Nutrition and Exercise Metabolism, 22(4), 251-256. doi:10.1123/ijsnem.22.4.251

Boorsma, R.K., Whitfield, J., \& Spriet, L.L. (2014). Beetroot juice supplementation does not improve performance of elite $1500-\mathrm{m}$ 
runners. Medicine \& Science in Sports \& Exercise, 46(12), 23262334. PubMed ID: 24781895 doi:10.1249/MSS.0000000000000364

Bosquet, L., Duchene, A., Dupont, G., Leger, L., \& Carter, H. (2007). VO2 kinetics during supramaximal exercise: Relationship with oxygen deficit and 800-m running performance. International Journal of Sports Medicine, 28(6), 518-524. PubMed ID: 17357962 doi:10. 1055/s-2006-955896

Bourdillon, N., Fan, J.L., Uva, B., Müller, H., Meyer, P., \& Kayser, B. (2015). Effect of oral nitrate supplementation on pulmonary hemodynamics during exercise and time trial performance in normoxia and hypoxia: A randomized controlled trial. Frontiers in Physiology, 6, 288. PubMed ID: 26528189 doi:10.3389/fphys. 2015.00288

Cermak, N.M., Gibala, M.J., \& van Loon, L.J. (2012). Nitrate supplementation's improvement of 10-km time-trial performance in trained cyclists. International Journal Sports Nutrition and Exercise Metabolism, 22(1), 64-71. doi:10.1123/ijsnem.22.1.64

Chapman, R.F., Karlsen, T., Resaland, G.K., Ge, R.L., Harber, M.P., Witkowski, S., ... Levine, B.D. (2014). Defining the "dose" of altitude training: How high to live for optimal sea level performance enhancement. Journal of Applied Physiology, 116(6), 595-603. PubMed ID: 24157530 doi:10.1152/japplphysiol.00634.2013

Christensen, P.M., Nyberg, M., \& Bangsbo, J. (2013). Influence of nitrate supplementation on $\mathrm{VO}_{2}$ kinetics and endurance of elite cyclists. Scandinavian Journal of Medicine and Science in Sports, 23(1), e21-e31. PubMed ID: 23020760 doi:10.1111/sms.12005

Cocksedge, S.P., Breese, B.C., Morgan, P.T., Nogueira, L., Thompson, C., Wylie, L.J., .. Bailey, S.J. (2020). Influence of muscle oxygenation and nitrate-rich beetroot juice supplementation on $\mathrm{O} 2$ uptake kinetics and exercise tolerance. Nitric Oxide, 99, 25-33. PubMed ID: 32272260 doi:10.1016/j.niox.2020.03.007

Goodall, S., Ross, E.Z., \& Romer, L.M. (2010). Effect of graded hypoxia on supraspinal contributions to fatigue with unilateral knee-extensor contractions. Journal of Applied Physiology, 109(6), 1842-1851. PubMed ID: 20813979 doi:10.1152/ japplphysiol.00458.2010

Jones, A.M., Ferguson, S.K., Bailey, S.J., Vanhatalo, A., \& Poole, D.C. (2016). Fiber type-specific effects of dietary nitrate. Exercise and Sport Sciences Reviews, 44(2), 53-60. PubMed ID: 26829247 doi:10. 1249/JES.0000000000000074

Jones, A.M., Thompson, C., Wylie, L.J., \& Vanhatalo, A. (2018). Dietary nitrate and physical performance. Annual Review of Nutrition, 38(1), 303-328. PubMed ID: 30130468 doi:10.1146/annurev-nutr-082117051622

Kelly, J., Vanhatalo, A., Bailey, S.J., Wylie, L.J., Tucker, C., List, S., . . Jones, A.M. (2014). Dietary nitrate supplementation: Effects on plasma nitrite and pulmonary $\mathrm{O}_{2}$ uptake dynamics during exercise in hypoxia and normoxia. American Journal of Physiology. Regulatory Integrative and Comparative Physiology, 307(7), R920-R930. doi:10.1152/ajpregu.00068.2014

Kent, G.L., Dawson, B., McNaughton, L.R., Cox, G.R., Burke, L.M., \& Peeling, P. (2019). The effect of beetroot juice supplementation on repeat-sprint performance in hypoxia. Journal of Sports Science, 37(3), 339-346. doi:10.1080/02640414.2018.1504369

MacLeod KE, Nugent SF, Barr SI, Koehle MS, Sporer BC, \& MacInnis MJ. (2015). Acute beetroot juice supplementation does not improve cycling performance in normoxia or moderate hypoxia. International Journal Sports Nutrition and Exercise Metabolism, 25(4), 359-366. doi:10.1123/ijsnem.2014-0129

Masschelein, E., Van Thienen, R., Wang, X., Van Schepdael, A., Thomis, M., \& Hespel, P. (2012). Dietary nitrate improves muscle but not cerebral oxygenation status during exercise in hypoxia. Journal of
Applied Physiology, 113(5), 736-745. PubMed ID: 22773768 doi:10. 1152/japplphysiol.01253.2011

Muggeridge, D.J., Howe, C.C., Spendiff, O., Pedlar, C., James, P.E., \& Easton, C. (2013). The effects of a single dose of concentrated beetroot juice on performance in trained flatwater kayakers. International Journal Sports Nutrition and Exercise Metabolism, 23(5), 498506. doi:10.1123/ijsnem.23.5.498

Muggeridge, D.J., Howe, C.C., Spendiff, O., Pedlar, C., James, P.E., \& Easton, C. (2014). A single dose of beetroot juice enhances cycling performance in simulated altitude. Medicine \& Science in Sports \& Exercise, 46(1), 143-150. PubMed ID: 23846159 doi:10.1249/MSS. 0b013e3182a1dc51

Nyakayiru, J., Jonvik, K.L., Trommelen, J., Pinckaers, P.J., Senden, J.M., van Loon, L.J., \& Verdijk, L.B. (2017). Beetroot juice supplementation improves high-intensity intermittent type exercise performance in trained soccer players. Nutrients, 9(3), 314. doi:10.3390/ nu9030314

Nybäck, L., Glännerud, C., Larsson, G., Weitzberg, E., Shannon, O.M., \& McGawley, K. (2017). Physiological and performance effects of nitrate supplementation during roller-skiing in normoxia and normobaric hypoxia. Nitric Oxide, 70, 1-8. PubMed ID: 28782598 doi:10. 1016/j.niox.2017.08.001

Pawlak-Chaouch, M., Boissière, J., Munyaneza, D., Gamelin, F.X., Cuvelier, G., Berthoin, S., \& Aucouturier, J. (2019). Beetroot juice does not enhance supramaximal intermittent exercise performance in elite endurance athletes. Journal of the American College of Nutrition, 38(8), 729-738. PubMed ID: 31084516 doi:10.1080/07315724. 2019.1601601

Porcelli, S., Ramaglia, M., Bellistri, G., Pavei, G., Pugliese, L., Montorsi, M., ... Marzorati, M. (2015). Aerobic fitness affects the exercise performance responses to nitrate supplementation. Medicine \& Science in Sports \& Exercise, 47(8), 1643-1651. PubMed ID: 25412295 doi:10.1249/MSS.0000000000000577

Rokkedal-Lausch, T., Franch, J., Poulsen, M.K., Thomsen, L.P., Weitzberg, E., Kamavuako, E.N., ... Larsen, R.G. (2019). Chronic high-dose beetroot juice supplementation improves time trial performance of welltrained cyclists in normoxia and hypoxia. Nitric Oxide, 85, 44-52. PubMed ID: 30685420 doi:10.1016/j.niox.2019.01.011

Shannon, O.M., Barlow, M.J., Duckworth, L., Williams, E., Wort, G., Woods, D., ... O'Hara, J.P. (2017). Dietary nitrate supplementation enhances short but not longer duration running time-trial performance. European Journal of Applied Physiology, 117(4), 775-785. PubMed ID: 28251402 doi:10.1007/s00421-017-3580-6

Shannon, O.M., Duckworth, L., Barlow, M.J., Woods, D., Lara, J., Siervo, M., \& O'Hara, J.P. (2016). Dietary nitrate supplementation enhances high-intensity running performance in moderate normobaric hypoxia, independent of aerobic fitness. Nitric Oxide, 59, 63-70. PubMed ID: 27553127 doi:10.1016/j.niox.2016.08.001

Stellingwerff, T., Peeling, P., Garvican-Lewis, L.A., Hall, R., Koivisto, A.E., Heikura, I.A., \& Burke, L.M. (2019). Nutrition and altitude: Strategies to enhance adaptation, improve performance and maintain health: A narrative review. Sports Medicine, 49(Suppl. 2), 169-184. PubMed ID: 31691928 doi:10.1007/s40279-019-01159-w

Thompson, C., Vanhatalo, A., Jell, H., Fulford, J., Carter, J., Nyman, L., ... Jones, A.M. (2016). Dietary nitrate supplementation improves sprint and high-intensity intermittent running performance. Nitric Oxide, 61, 55-61. PubMed ID: 27777094 doi:10.1016/j.niox.2016. 10.006

Wickham, K.A., \& Spriet, L.L. (2019). No longer beeting around the bush: A review of potential sex differences with dietary nitrate supplementation. Applied Physiology Nutrition and Metabolism, 44(9), 915-924. doi:10.1139/apnm-2019-0063 
Wylie, L.J., Bailey, S.J., Kelly, J., Blackwell, J.R., Vanhatalo, A., \& Jones, A.M. (2016). Influence of beetroot juice supplementation on intermittent exercise performance. European Journal of Applied Physiology, 116(2), 415-425. PubMed ID: 26614506 doi:10.1007/ s00421-015-3296-4

Wylie, L.J., Kelly, J., Bailey, S.J., Blackwell, J.R., Skiba, P.F., Winyard, P.G., ... Jones, A.M. (2013a). Beetroot juice and exercise:
Pharmacodynamic and dose-response relationships. Journal of Applied Physiology, 115(3), 325-336. doi:10.1152/japplphysiol.00372.2013

Wylie, L.J., Mohr, M., Krustrup, P., Jackman, S.R., Ermıdis, G., Kelly, J., . . Jones, A.M. (2013b). Dietary nitrate supplementation improves team sport-specific intense intermittent exercise performance. European Journal of Applied Physiology, 113(7), 1673-1684. doi:10. 1007/s00421-013-2589-8 